\title{
ELECTROCHEMICAL PROTEIN CLEAVAGE IN A MICROFLUIDIC CELL WITH INTEGRATED BORON DOPED DIAMOND ELECTRODES
}

Floris T.G. van den Brink, ${ }^{\mathrm{a} \dagger *}$ Tao Zhang, ${ }^{\mathrm{b} \dagger}$ Liwei Ma, ${ }^{\mathrm{a}}$ Johan Bomer, ${ }^{\mathrm{a}}$ Mathieu Odijk, ${ }^{\mathrm{a}}$ Wouter Olthuis, ${ }^{\mathrm{a}}$ Hjalmar P. Permentier, ${ }^{b}$ Rainer Bischoff ${ }^{b}$ and Albert van den Berg ${ }^{a}$

${ }^{\text {a }}$ BIOS - Lab on a Chip group, MESA+ Institute for Nanotechnology and MIRA Institute for Biomedical Technology and Technical Medicine, University of Twente, 7500 AE Enschede, The Netherlands

${ }^{b}$ Analytical Biochemistry and Interfaculty Mass Spectrometry Center, Department of Pharmacy, University of Groningen, 9713 AV Groningen, The Netherlands

$\dagger$ These authors contributed equally.

*E-mail: f.t.g.vandenbrink@utwente.nl.

\section{CONTENTS}

S1. Sample preparation, data acquisition and database searching …........................................2

S2. Hydraulic resistance of the frit channel network............................................................ 3

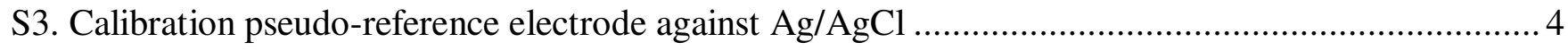

S4. Electrochemical oxidation and cleavage mechanisms of LWL and LYL ................................5

S5. LC-MS extracted ion chromatograms of oxidized and cleaved LWL.................................... 6

S6. LC-MS extracted ion chromatograms of oxidized and cleaved LYL . ................................... 7

S7. LC-MS extracted ion chromatograms of oxidized and cleaved LFL ..................................... 8

S8. LC-MS extracted ion chromatograms of ACTH cleavage products ........................................ 9

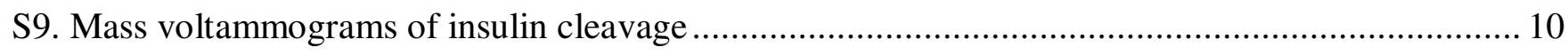

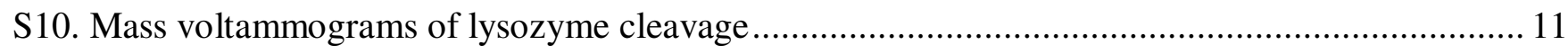

S11. LC-MS extracted ion chromatograms of lysozyme cleavage products ................................. 12 


\section{S1. SAMPLE PREPARATION, DATA ACQUISITION AND DATABASE SEARCHING}

\section{Sample preparation}

Stock solutions of LWL, LYL, LFL and ACTH 1-10 were prepared in 89/10/1 (v/v/v) water/acetonitrile/formic acid at a concentration of 1 $\mathrm{mM}$. Solutions of lysozyme and insulin were prepared in 89/10/1 (v/v/v) water/acetonitrile/formic acid to a protein concentration of 100 $\mu \mathrm{M}$. For reduction and alkylation of lysozyme, $100 \mu \mathrm{M}$ protein was prepared in $1 \mathrm{~mL}$ ammonium bicarbonate buffer (100 mM, pH 8). DTT was added at a concentration of $2 \mathrm{mM}$ and incubated for $30 \mathrm{~min}$ at $60^{\circ} \mathrm{C}$. IAM was added at a concentration of $20 \mathrm{mM}$ after cooling and incubated in a dark environment at room temperature for $60 \mathrm{~min}$. After alkylation, $8 \mathrm{mM}$ DTT was added and incubated in a dark environment at room temperature for $30 \mathrm{~min}$ to quench the alkylation reaction. Lysozyme precipitated upon reduction and alkylation. The reaction mixture was centrifuged at $13000 \mathrm{rpm}$ and the supernatant was removed. $1 \mathrm{~mL} \mathrm{89/10/1} \mathrm{(v/v/v)} \mathrm{water/acetonitrile/formic} \mathrm{acid} \mathrm{was}$ added to the precipitate to prepare a $100 \mu \mathrm{M}$ stock solution of reduced and alkylated lysozyme.

Prior to electrochemical oxidation and cleavage, the peptides and proteins were diluted to a final concentration of $10 \mu \mathrm{M}(\mathrm{LWL}, \mathrm{LYL}$, LFL, ACTH, insulin) or $2 \mu \mathrm{M}$ (lysozyme). To prevent acid hydrolysis or formylation of the proteins during storage, the formic acid content of peptide and protein samples was increased to $5 \%$ just before the electrochemistry experiments.

\section{Data acquisition}

MS scans from $\mathrm{m} / z, 100$ to 1500 (LWL, LYL and LFL and ACTH 1-10) and MS scans from $\mathrm{m} / \mathrm{z} 200$ to 2000 (insulin and lysozyme) were recorded at a resolution of 72000 and MS/MS spectra were recorded at a resolution of 17500 after fragmentation in the high-energy collisional dissociation (HCD) cell. The normalized collision energy was set at $35 \mathrm{~V}$. All data was acquired in profile mode using positive polarity.

\section{Data analysis and database searching}

The LC-MS/MS data were analyzed with the database search engine PEAKS (version 7.5, Bioinformatics Solutions Inc) using the SwissProt database of (chicken) containing 2601 protein sequences. The search parameters were as follows: Parent Mass Error Tolerance: 10.0 ppm; Fragment Mass Error Tolerance: 0.7 Da; Enzyme: EC (custom-defined, digestion after Y or W); Max Missed Cleavages: 5; Non-specific Cleavage: none; Variable Modifications: Oxidation (on MFWHYC): +15.99, Carbamidomethylation (C): +57.02; EC-Y-2 (custom-defined: on C-terminal Y): -2.02; EC-W+14 (custom-defined: on C-terminal W): +13.98 ; Maximum variable post-translational modifications per peptide: 5 . The false discovery rate (FDR) threshold was set to $0.1 \%$ on the peptide level. 


\section{S2. HYDRAULIC RESISTANCE OF THE FRIT CHANNEL NETWORK}

The hydraulic resistance of rectangular channels with $w>h$, filled with a liquid having a dynamic viscosity $\mu$, can be approximated by: ${ }^{1}$

$$
R_{h y} \approx \frac{12 \mu l}{w h^{3}\left(1-0.63\left(\frac{h}{w}-\frac{h^{5}}{12 w^{5}}\right)\right)}
$$

Since $1 / R_{h y}$ scales with the third power of $h$ (discarding higher order terms), whereas $1 / R_{e l}$ scales linear with the channel height (equation 3 in the main text), having an array of shallow and narrow frit channels helps in limiting convection through the frit channel network, while maintaining sufficient conductance. In addition, the double branches of frit channels in each corner of the meandering WE and CE channels limit undesired convection through the frit channel network as a result of the pressure gradient in the direction of the flow. The hydraulic resistance of a frit channel ladder can be calculated using the hydraulic resistance of a frit channel $\left(R_{\text {frit }}, h y\right)$ combined with the hydraulic resistances of the pieces of connecting channel $\left(R_{c c, h y}\right)$ and working electrode channel $\left(R_{w, h y}\right)$ between adjacent frit channels. Using an approach analogous to equation 5 in the main text, we find:

$$
R_{l, h y}=\left(\sum_{i=1}^{N} \frac{1}{R_{\text {frit }, h y}+(i-1) R_{c c, h y}+(N-i) R_{w, h y}}\right)^{-1}
$$

Taking into account the interconnecting channel $\left(R_{i c, h y}\right)$, the hydraulic resistance of a frit channel branch can be calculated as:

$$
R_{b, h y}=2 R_{l, h y}+R_{i c, h y}
$$

Prior to the cell outlets, hydraulic resistors are included to equalize the resistance of the WE and CE channels, providing the same flow rate in both channels and thereby reducing the risk of convection in the frit channel network. Unless a large external hydraulic resistance is coupled to one of the channel outlets, the ratio of flow rates in both channels will be mainly determined by these structures.

To illustrate the use of these hydraulic resistors, one can consider the outlet region of the cell, starting from the last frit channel branch, via the hydraulic resistors to the outlets (figure 1D in the main text). Both WE and CE channels are supplied with a constant flow rate $Q_{i n}$, which is half the total flow rate, and the hydraulic resistance of the channel structure between frit channels and outlet, including the flow resistor, is designated $R_{f, h y}$. Upon a pressure variation at one of the outlets $(\Delta P)$, an undesired flow through the frit channel branch $\left(Q_{f b}\right)$ can be expected to occur, which is in this simplified situation equal to:

$$
Q_{f b}=\frac{\Delta P}{2 R_{f, h y}+R_{b, h y}}
$$

If, for example, capillary tubing with a length of $0.5 \mathrm{~m}$ and $100 \mu \mathrm{m}$ inner diameter is connected to one outlet only, while $Q_{i n}$ is maintained at $1 \mu \mathrm{L} / \mathrm{min}$, a flow rate $Q_{f b}$ of $16 \mathrm{~nL} / \mathrm{min}$ could be expected in this frit channel branch. In comparison, this unbalanced load will cause a flow of $43 \mathrm{~nL} / \mathrm{min}$ through the frit channel branch in the absence of hydraulic resistors. Therefore, by integrating these simple structures one can connect an asymmetrical load with a ratio that is $\sim 3$ times higher before seeing the undesired effects of asymmetrical flow rates to the same extent as one would see when these hydraulic resistors are absent. 


\section{S3. CALIBRATION PSEUDO-REFERENCE ELECTRODE AGAINST AG/AGCL}

To be able to relate the protein cleavage potentials in the microfluidic electrochemical cell to a $\mathrm{KCl}$ saturated $\mathrm{Ag} / \mathrm{AgCl}$ reference electrode, the Pt pRE was calibrated against $\mathrm{Ag} / \mathrm{AgCl}$ in the two solutions containing 89/10/1 (v/v/v) water/acetonitrile/formic acid (pH 2.0) and $85 / 10 / 5(\mathrm{v} / \mathrm{v} / \mathrm{v})$ water/acetonitrile/formic acid ( $\mathrm{pH} 1.5)$, respectively. Cyclic voltammograms of $1 \mathrm{mM} 1,1$ '-ferrocenedimethanol in these two solutions were recorded in both the regular electrochemical cell (micro-structured BDD WE, platinum $\mathrm{CE}$ and saturated Ag/AgCl RE) and the microfluidic electrochemical cell (BDD WE and CE, Pt pRE) and the half-wave potential from the second scan of each CV was determined in both systems (see CVs in figure S1). From these measurements, the potential of the platinum pRE was determined to be on average $(\mathrm{n}=3) 225 \mathrm{mV}$ vs. $\mathrm{Ag} / \mathrm{AgCl}(\mathrm{KCl}$ saturated) in the $1 \%$ formic acid solution and on average $(\mathrm{n}=3) 196 \mathrm{mV} \mathrm{vs} . \mathrm{Ag} / \mathrm{AgCl}(\mathrm{KCl}$ saturated) in the $5 \%$ formic acid solution.

A $1 \mathrm{mM} 1,1^{\prime}$-ferrocenedimethanol in 89/10/1 DI/ACN/formic acid

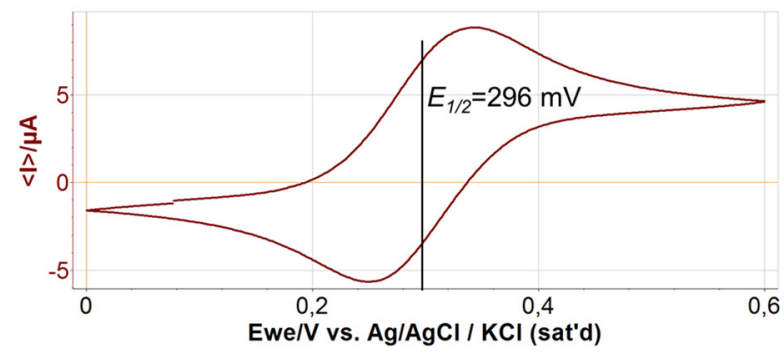

C $1 \mathrm{mM} \mathrm{1,1'-ferrocenedimethanol} \mathrm{in} \mathrm{89/10/1} \mathrm{DI/ACN/formic} \mathrm{acid}$

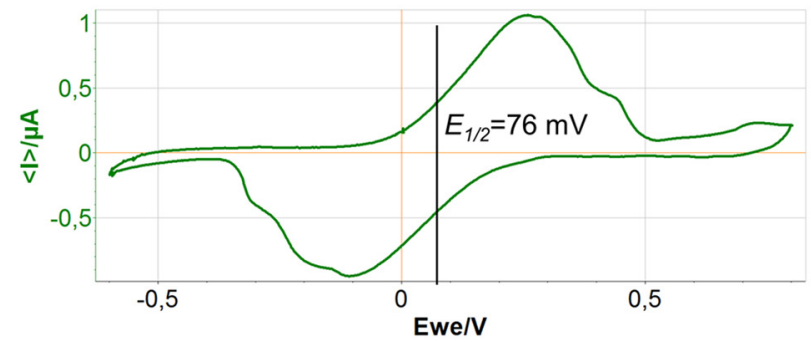

B $1 \mathrm{mM}$ 1,1'-ferrocenedimethanol in 85/10/5 DI/ACN/formic acid

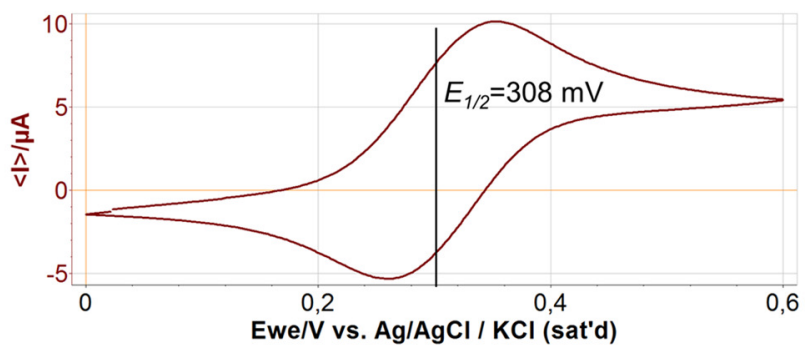

D $1 \mathrm{mM} 1,1$ '-ferrocenedimethanol in 85/10/5 DI/ACN/formic acid

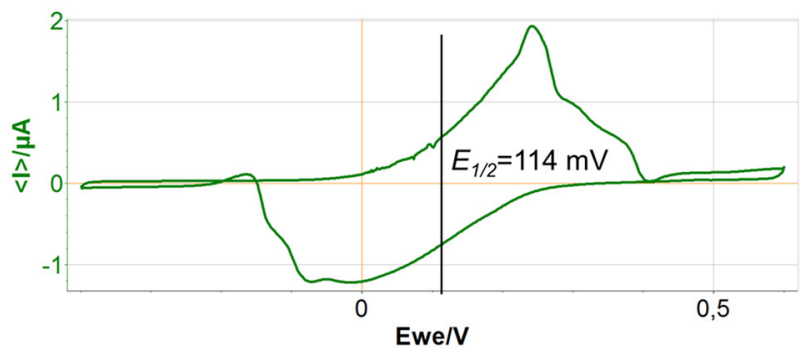

Figure S3: Cyclic voltammograms recorded using $1 \mathrm{mM} \mathrm{1,1'-ferrocenedimethanol} \mathrm{in} \mathrm{the} \mathrm{two} \mathrm{solutions} \mathrm{used} \mathrm{throughout} \mathrm{the} \mathrm{experiments}$ (10\% acetonitrile in $1 \%$ and $5 \%$ formic acid). A and B: voltammograms recorded using a regular electrochemical cell with a BDD working electrode (WE), platinum counter electrode (CE) and $\mathrm{Ag} / \mathrm{AgCl}(\mathrm{KCl}$ saturated) reference electrode (RE). C and $\mathrm{D}$ : voltammograms recorded using a microfluidic electrochemical cell with a BDD WE and CE and a platinum pseudo-RE. 
S4. ELECTROCHEMICAL OXIDATION AND CLEAVAGE MECHANISMS OF LWL AND LYL

A
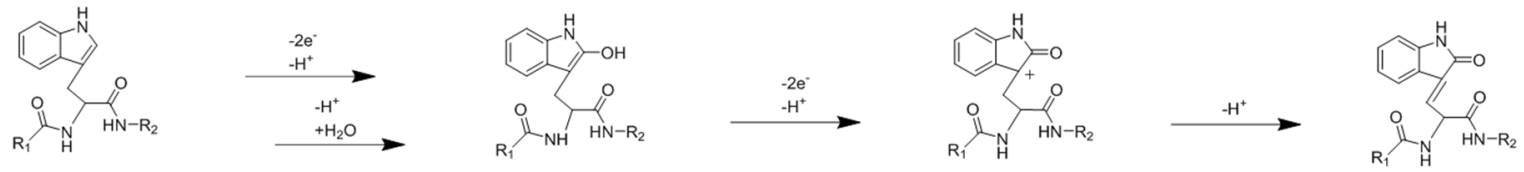

M

$M+16$

$M+14$
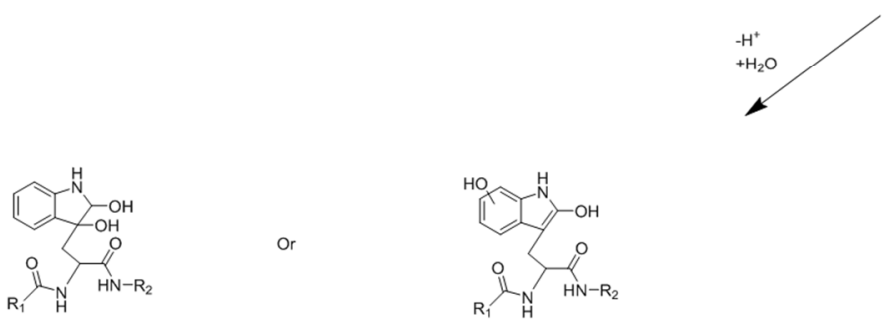

$\mathrm{M}+32$

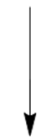

Or

$M+32$
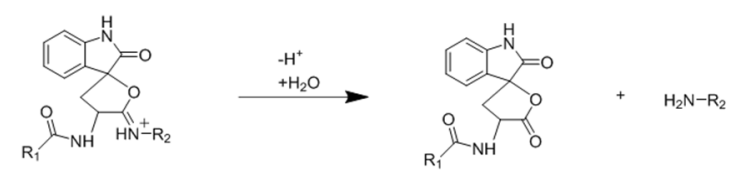

$R_{1} W+14$

B
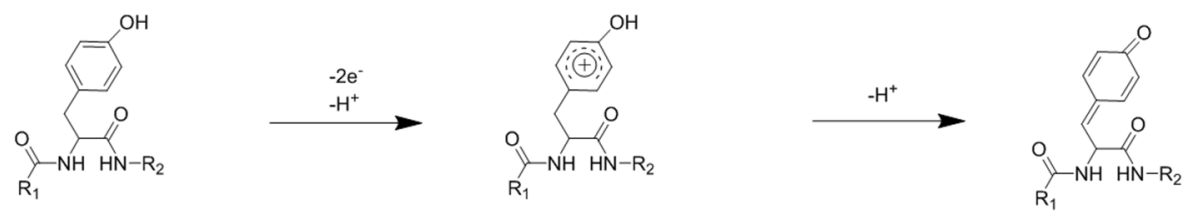

M

M-2
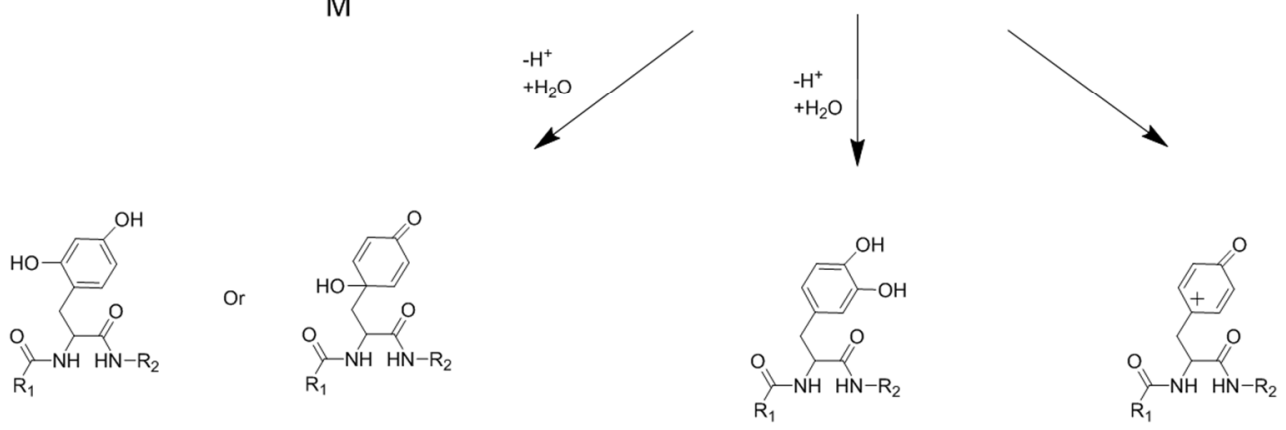

$M+16$

$M+16$
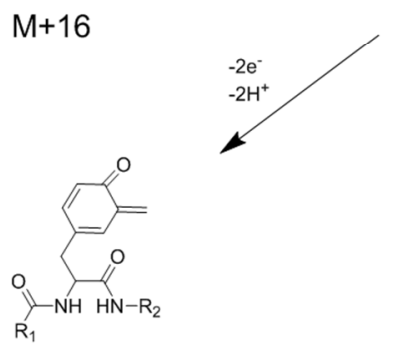

$M+16$

$\mathrm{M}+14$
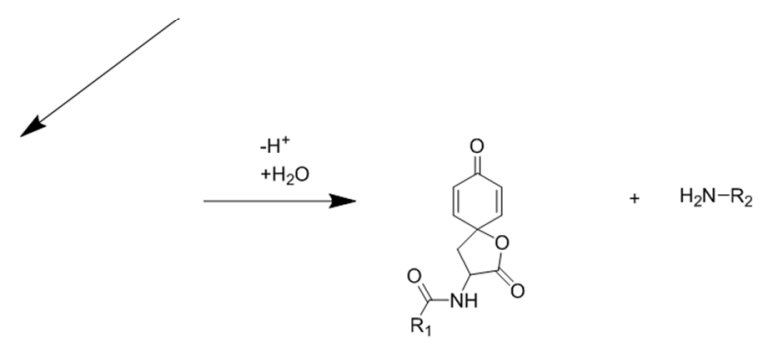

$\mathrm{R}_{1} \mathrm{Y}-2$

Scheme S4: Proposed electrochemical oxidation and cleavage pathways of A: tryptophan-containing peptides and proteins and B: tyrosine-containing peptides and proteins. Adapted with permission from reference 14. Copyright 2010 American Chemical Society. 


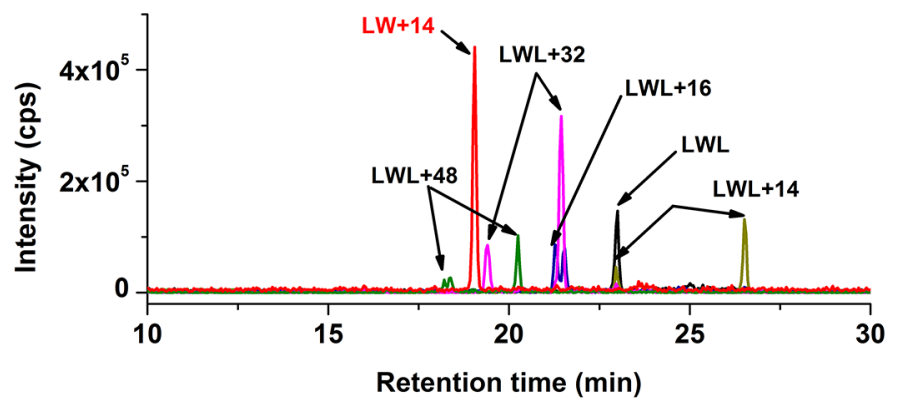

Figure S5: LC-MS extracted ion chromatograms of products collected from a microfluidic electrochemical cell after oxidation and cleavage of $10 \mu \mathrm{M} \mathrm{LWL}$ in 89/10/1 (v/v/v) water/acetonitrile/formic acid at a potential of $1300 \mathrm{mV}$ at a BDD working electrode. Analyte was introduced at a total flow rate of $2 \mu \mathrm{L} / \mathrm{min}(1 \mu \mathrm{L} / \mathrm{min}$ over the $\mathrm{WE})$. A number of oxidation products are observed in addition to the cleavage product $\mathrm{LW}+14$. 


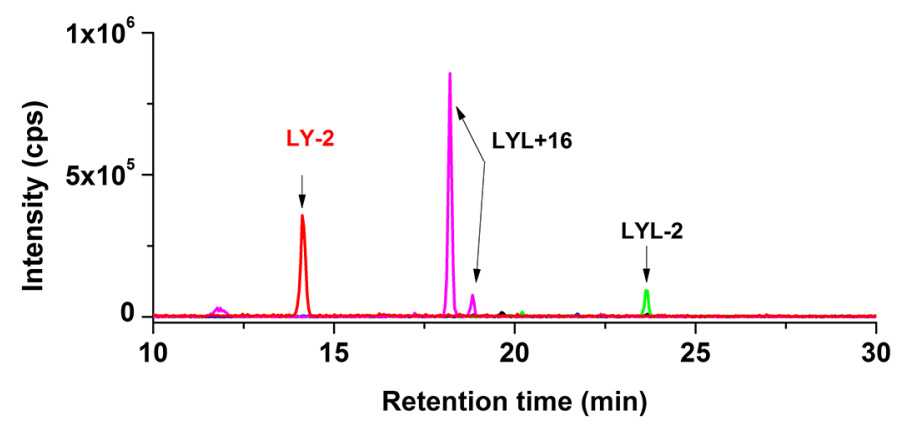

Figure S6: LC-MS extracted ion chromatograms of products collected from a microfluidic electrochemical cell after oxidation and cleavage of $10 \mu \mathrm{M} \mathrm{LYL}$ in 89/10/1 (v/v/v) water/acetonitrile/formic acid at a potential of $2000 \mathrm{mV}$ at a BDD working electrode. Analyte was introduced at a total flow rate of $2 \mu \mathrm{L} / \mathrm{min}(1 \mu \mathrm{L} / \mathrm{min}$ over the $\mathrm{WE})$. A number of oxidation products are observed in addition to the cleavage product LY-2. 

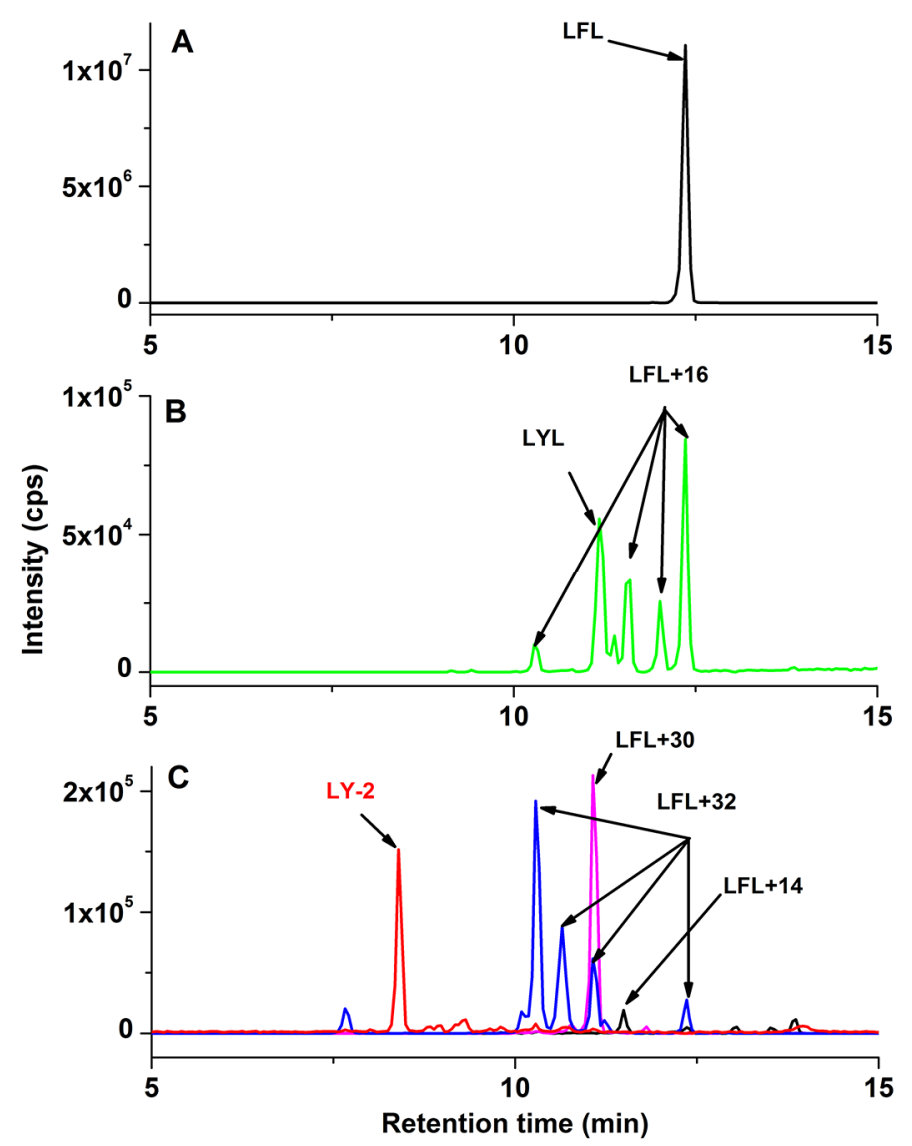

Figure S7: LC-MS extracted ion chromatograms of the products collected from a microfluidic electrochemical cell after oxidation and cleavage of $10 \mu \mathrm{M} \mathrm{LFL}$ in 85/10/5 (v/v/v) water/acetonitrile/formic acid at $2000 \mathrm{mV}$ at a BDD working electrode. Analyte was introduced at a total flow rate of $2 \mu \mathrm{L} / \mathrm{min}(1 \mu \mathrm{L} /$ min over the WE). A: starting material LFL; B: oxidation (aromatic hydroxylation) products LFL+16 including LYL and C: further oxidation products LFL+14, LFL+30, LFL+32 and the cleavage product LY-2. 


\section{ACTH 1-10 (SYSMEHFRWG)}

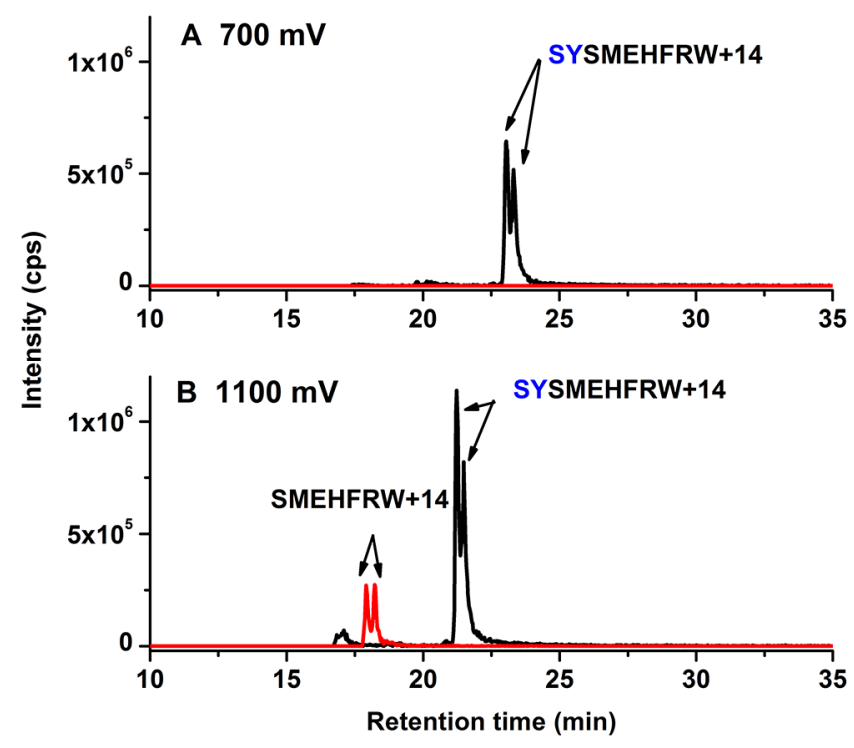

Figure S8: LC-MS extracted ion chromatograms of cleavage products of ACTH 1-10 (10 $\mu \mathrm{M}$ in 89/10/1 (v/v/v) water/acetonitrile/formic acid) generated at the BDD working electrode in a microfluidic electrochemical cell. Analyte was introduced at a total flow rate of 2 $\mu \mathrm{L} / \mathrm{min}(1 \mu \mathrm{L} / \mathrm{min}$ over the WE). A: cleavage products generated at $700 \mathrm{mV}$ and B: cleavage products generated at $1100 \mathrm{mV}$. A pair of two isobaric chromatographic peaks with identical MS and MS/MS spectra were observed for each cleavage product, and the nature of these compounds is currently under investigation. 


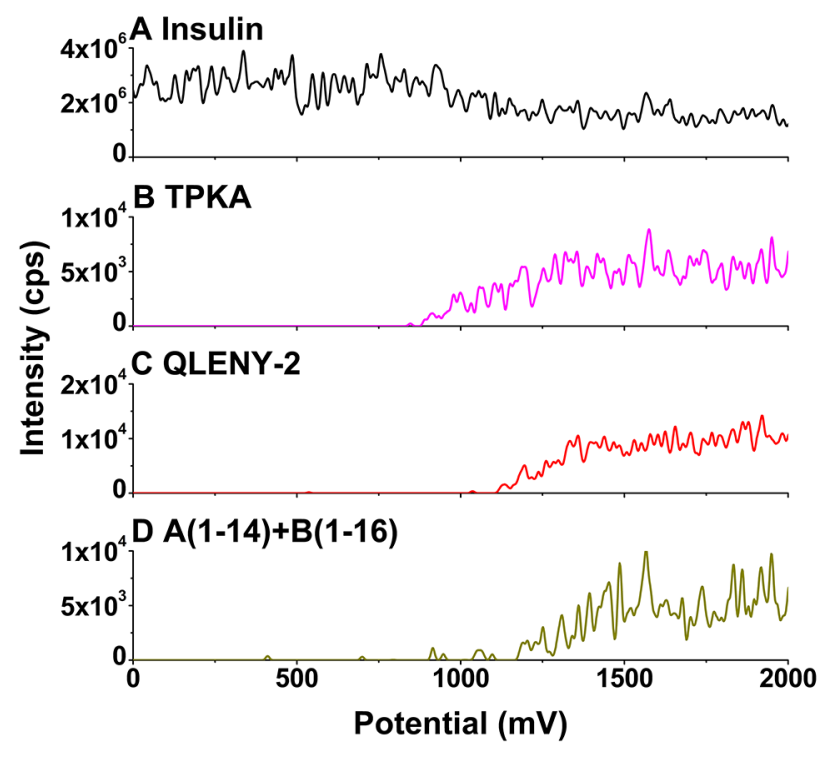

Figure S9: Mass voltammograms of $10 \mu \mathrm{M}$ insulin in 85/10/5 (v/v/v) water/acetonitrile/formic acid. The potential was ramped from 0 to $2000 \mathrm{mV}$ at a scan rate of $2 \mathrm{mV} / \mathrm{s}$ while analyte was introduced at a total flow rate of $2 \mu \mathrm{L} / \mathrm{min}(1 \mu \mathrm{L} / \mathrm{min}$ over the BDD WE). Traces of insulin and its cleavage products were plotted as a function of cell potential. A: Insulin $(\mathrm{m} / z \mathrm{z} 1146.93$, charge 5+); B: TPKA $(\mathrm{m} / z$, 208.63, charge 2+); C: QLENY-2 ( $\mathrm{m} / \mathrm{z}$ 664.29, charge $1+)$ and D: $\mathrm{A}(1-14)+\mathrm{B}(1-16)(\mathrm{m} / \mathrm{z}$ 1099.16, charge $3+)$. $\mathrm{A}(1-14)+\mathrm{B}(1-16)$ corresponds to the $\mathrm{N}$-terminal parts of the A- and B-chains released upon cleavage at sites 1 and 3, respectively, which remain linked together by a disulfide bond (see figure 5 in the main text for details). 
S10. MASS VOLTAMMOGRAMS OF LYSOZYME CLEAVAGE

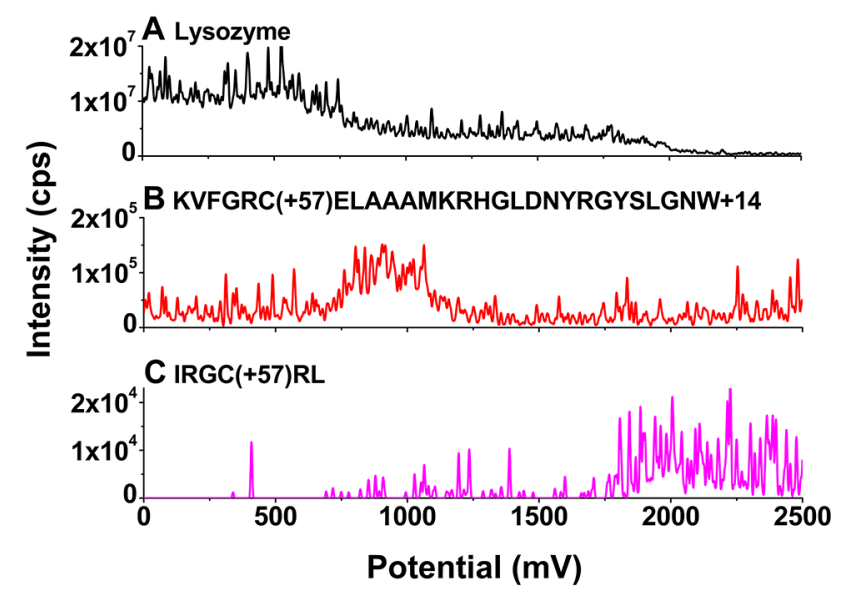

Figure S10: Mass voltammograms of $2 \mu \mathrm{M}$ lysozyme in 85/10/5 (v/v/v) water/acetonitrile/formic acid. The potential was ramped from 0 to $2500 \mathrm{mV}$ at a scan rate of $2 \mathrm{mV} / \mathrm{s}$ while analyte was introduced at a total flow rate of $2 \mu \mathrm{L} / \mathrm{min}(1 \mu \mathrm{L} / \mathrm{min}$ over the BDD WE). Traces for lysozyme and two products (KVFGRC(+57)ELAA-AMKRHGLDNY-RGYSLGNW+14 and IRGC(+57)RL) resulting from cleavage at the C-terminal of tryptophan (sites 3 and 9) were extracted and plotted versus cell potential. A: Lysozyme $(\mathrm{m} / z$. 739.37, charge $20+)$, B: KVFGRC(+57)ELAA-AMKRHGLDNY-RGYSLGNW+14 ( $\mathrm{m} / \mathrm{z}$ 657.53, charge 5+) and C: IRGC(+57)RL ( $\mathrm{m} / \mathrm{z}$, 387.72, charge 2+). See table 1 in the main text for details. 


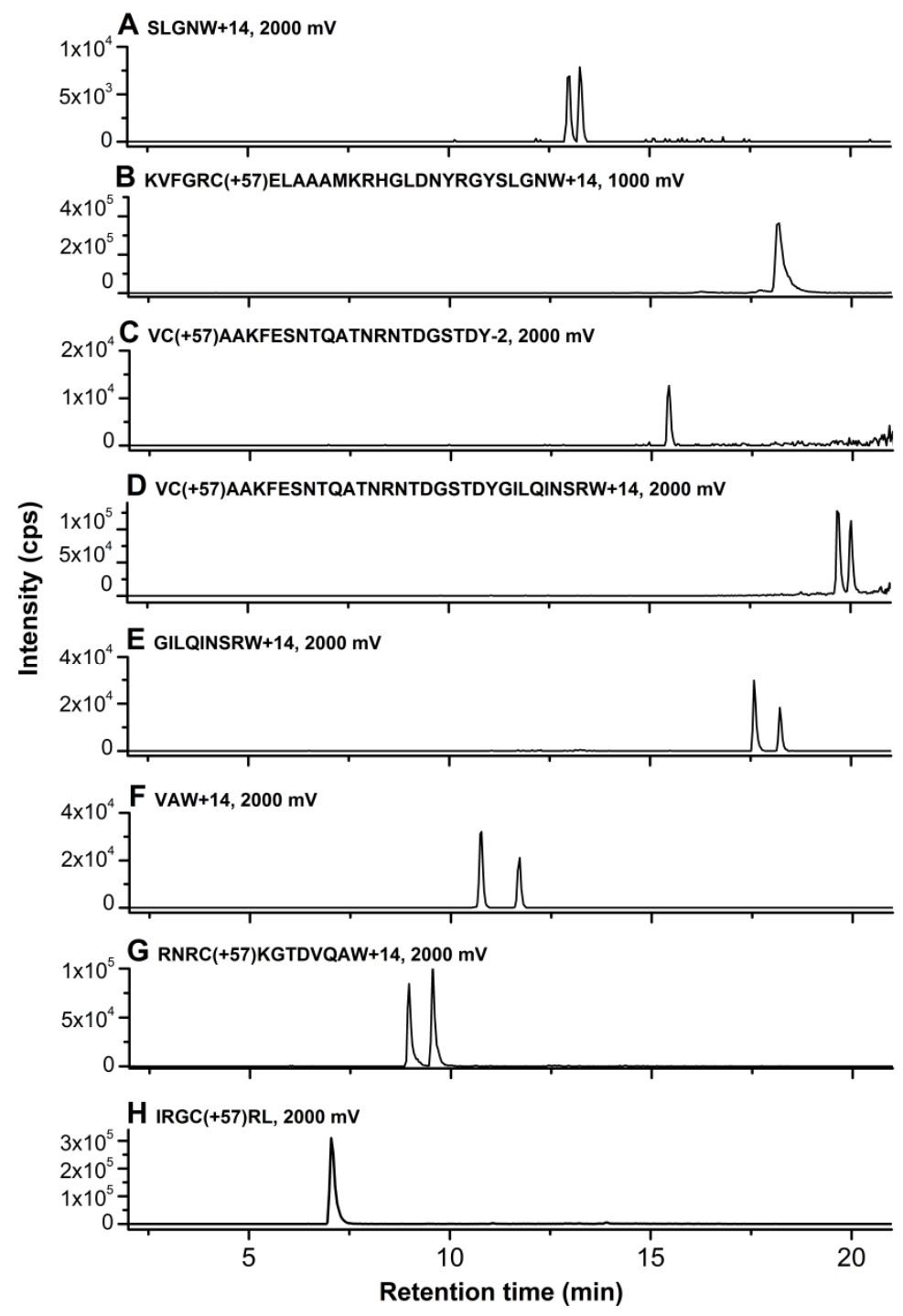

Figure S11: LC-MS extracted ion chromatograms of cleavage products of lysozyme ( $2 \mu \mathrm{M}$ in $85 / 10 / 5(\mathrm{v} / \mathrm{v} / \mathrm{v})$ water/acetonitrile/formic acid) generated at the BDD working electrode in a microfluidic electrochemical cell. Two different potentials were used (1000 and 2000 $\mathrm{mV})$ and analyte was introduced at a total flow rate of $2 \mu \mathrm{L} / \mathrm{min}(1 \mu \mathrm{L} / \mathrm{min}$ over the WE). See table 1 in the main text for details. Isobaric chromatographic peak pairs were observed for all 7 spirolactone-containing peptides with two exceptions, KVFGRC(+57)ELAAAMKRHGLDNY-RGYSLGNW+14 and VC(+57)AAKFESNF-NTQATNRNTD-GSTDY-2, which is possibly due to insufficient chromatographic separation. 